\title{
ChemComm
}

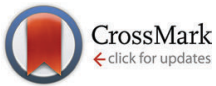

Cite this: Chem. Commun., 2016, 52,4298

Received 12th January 2016 Accepted 19th February 2016

DOI: $10.1039 / \mathrm{c} 6 \mathrm{cc00292g}$

www.rsc.org/chemcomm

\section{Surface-directed modulation of supramolecular gel properties $\dagger$}

\author{
Maria Galini Faidra Angelerou, ${ }^{a}$ Akmal Sabri, ${ }^{a}$ Rhiannon Creasey, ${ }^{b}$ \\ Polyxeni Angelerou, ${ }^{C}$ Maria Marlow*a and Mischa Zelzer*ad
}

\begin{abstract}
Supramolecular materials are widely studied and used for a variety of applications; in most applications, these materials are in contact with surfaces of other materials. Whilst much focus has been placed on elucidating factors that affect supramolecular material properties, the influence of the material surface on gel formation is poorly characterised. Here, we demonstrate that surface properties directly affect the fibre architecture and mechanical properties of self-assembled cytidine based gel films.
\end{abstract}

Supramolecular materials are under intense investigation in many areas such as conducting materials, energy and information storage, tissue engineering, sensors, coatings or catalysis ${ }^{1-3}$ due to their ability to self-heal, mimic biological functionalities and form structures with precise nano-scale order and interactions. ${ }^{4}$ The functionality and application potential of supramolecular materials is closely linked to their chemical, physical and mechanical properties which in turn are affected by processing conditions and gelation triggers such as concentration, $\mathrm{pH}$, temperature, solvent and enzymes. ${ }^{5-7}$

Among the factors affecting supramolecular self-assembly, the influence of the surrounding material surface on bulk gel properties has received little attention. The effect of surfaces on the formation of self-assembled monolayers is well established ${ }^{8}$ and a recent example demonstrated that the structure of a surface (i.e. graphite) can provide a template for monolayer formation and guide $2 \mathrm{D}$ self-assembly of molecules ( $p$-terphenyl-3,5, $3^{\prime \prime}, 5^{\prime \prime}$ tetracarboxylic acid) with matching dimensions. ${ }^{9}$

As surfaces can template and influence the self-assembly of monolayers we hypothesised that material surfaces may also

\footnotetext{
${ }^{a}$ University of Nottingham, School of Pharmacy, Nottingham NG7 2RD, UK. E-mail: pazmem@exmail.nottingham.ac.uk, pazmz@exmail.nottingham.ac.uk ${ }^{b}$ School of Chemistry, University of Queensland, St Lucia, 4067, Australia ${ }^{c}$ Delft University of Technology, School of Civil Engineering, 2628 CD, Delft, Netherlands

${ }^{d}$ National Physical Laboratory, Teddington, Middlesex, TW11 OLW, UK $\dagger$ Electronic supplementary information (ESI) available: Detailed experimental procedures, additional AFM and ToF-SIMS data and statistical analysis data. See DOI: $10.1039 / \mathrm{c} 6 \mathrm{cc} 00292 \mathrm{~g}$
}

have the potential to influence self-assembly of gelators and hence affect the properties of the resulting gels. To date the interplay between surface properties and bulk gelation is poorly understood and existing reports mostly focus on confinement of the self-assembly trigger to the surface. For example, it was shown that self-assembly can be triggered with surface immobilised enzymes to form fibres ${ }^{10}$ or electrochemically to form gel films. ${ }^{11}$ A direct influence of surface morphology on self-assembly was observed when diphenylalanine was placed in contact with either glass or a microporous mixed cellulose ester membrane, where the gelator formed nanofibres and microvesicles, respectively. ${ }^{7,12}$

Although the material surface has been recognised to play an important role in the self-assembly process ${ }^{13}$ the effect of this interaction on gel properties has not yet been demonstrated. Here, we report for the first time a direct relation between material surface properties and the physical properties of a gel film formed on the surface (Fig. 1).

To investigate the effect of surface properties on physical gel characteristics we formed gel films from one recently synthesized cytidine derivative, the $N-(1-((2 R, 4 S, 5 R)-4$-hydroxy-5-(hydroxymethyl)tetrahydrofuran-2-yl)-2-oxo-1,2-dihydropyrimidin-4-yl)octanamide (C14-cytidine $)^{14}$ on surfaces displaying two different chemical functionalities. The gelator is currently under investigation as a class of drug delivery system with low toxicity ${ }^{14}$ and we expect that its amphiphilic nature would enable it to interact differently with surfaces of high or low hydrophobicity.

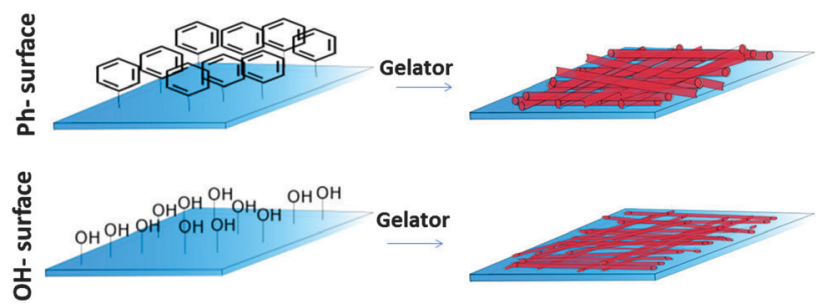

Fig. 1 Schematic of the experimental setup. Two surfaces (left) with different hydrophobicity were used as substrates for gel formation. After the application of the gelator on the substrate a fibrillar network was formed (right). 


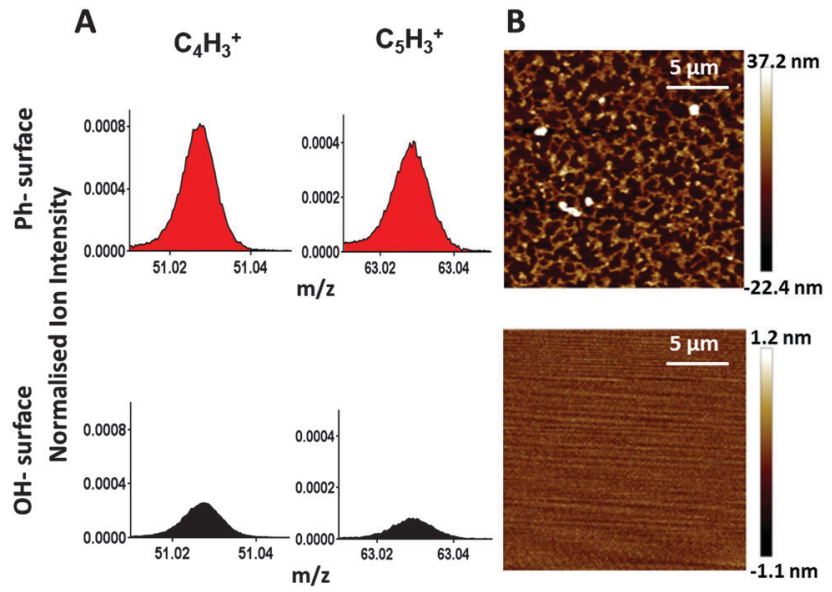

Fig. 2 Surface analysis on the $\mathrm{Ph}$-(top row) and the $\mathrm{OH}$-(bottom row) modified surfaces. (A) ToF-SIMS shows the presence of characteristic ions for phenyl groups on the Ph surfaces at $\mathrm{m} / z=51\left(\mathrm{C}_{4} \mathrm{H}_{3}{ }^{+}\right)$and $\mathrm{m} / \mathrm{z}=63$ $\left(\mathrm{C}_{5} \mathrm{H}_{3}{ }^{+}\right)$. Spectra were normalised to the total ion counts. (B) AFM height images showing different topographies on the $\mathrm{Ph}$ - and the $\mathrm{OH}$-surface.

Piranha cleaned glass was used as a polar surface (WCA = $24 \pm 2^{\circ}$ ) and silanisation of that glass surface with trimethoxyphenylsilane introduced phenyl groups providing a hydrophobic (WCA $=82 \pm 2^{\circ}$ ) surface. ToF-SIMS analysis confirmed the presence of phenyl-groups. The linear ion fragments $\mathrm{C}_{4} \mathrm{H}_{3}{ }^{+}$and $\mathrm{C}_{5} \mathrm{H}_{3}{ }^{+}$have previously been reported as characteristic ToF-SIMS ions from phenyl-surfaces ${ }^{15}$ and their intensity was significantly increased on the surface after silanisation (Fig. 2A), confirming chemical modification of the surface. The topography of the surfaces was measured by AFM (Fig. 2B). The OH-surface was smooth and featureless, while the $\mathrm{Ph}$-surface displayed a structured topography.

Gel films were formed on the two surfaces using the C14-cytidine (Fig. 3A) using an anti-solvent approach i.e., dissolving the gelators in ethanol and then adding the ethanol solution to water. The gelator is soluble in ethanol at the required concentration and temperature
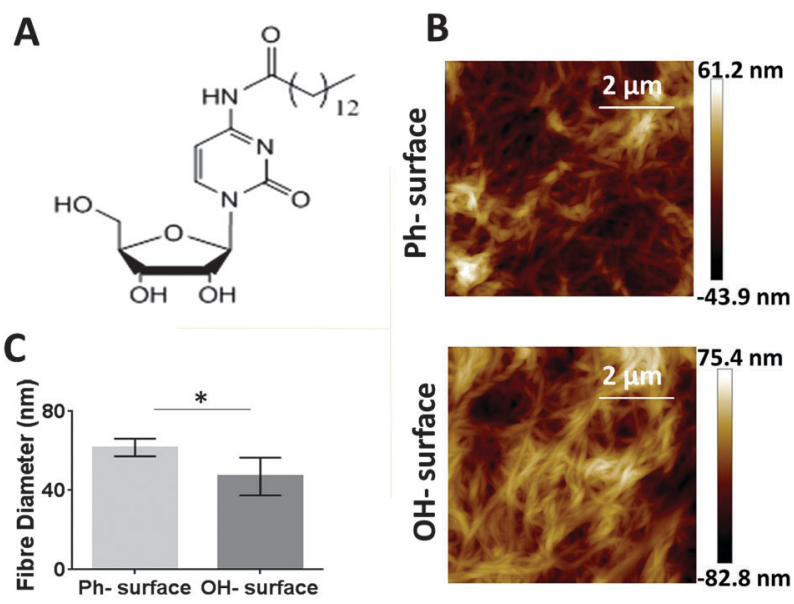

Fig. 3 The structure of C14-cytidine (A). AFM images of the gel structure formed by the two gelators on either the $\mathrm{OH}$ - or Ph-surface (B) and significantly different $(p<0.05)$ diameters of gel fibres determined from the AFM images (C). but insoluble in water. The gelator formed a fibrillar network (Fig. 3B). AFM data (see Fig. S1 in ESI $\dagger$ ) showed that on both surfaces the C14-cytidine formed a micrometer thick gel film $(1.0 \pm 0.2 \mu \mathrm{m})$.

In order to evaluate if the gel films are homogeneous over the whole sample, AFM images were taken at extreme points across the sample and the fibre diameters were determined (Fig. S3 in ESI $\dagger$ ). The gels' fibre diameters were found to be comparable at various locations on both surfaces, confirming that gelation occurred uniformly over the whole surface.

To establish if surface properties affect the physical and mechanical properties of the self-assembled cytidine gelator, AFM was used to measure the diameter of the self-assembled fibres as well as the Young's modulus of the gel film. When comparing the fibre diameter of both gelators on the two surfaces, significantly larger values were obtained on the $\mathrm{Ph}$-surface $(61.7 \pm 4.4 \mathrm{~nm})$ than on the OH-surface $(47 \pm 9 \mathrm{~nm})$ (Fig. 3C). This indicates a different interaction between the gelator molecules and the surface that ultimately leads to differences in the self-assembly pathway and

\section{A OH-surface}

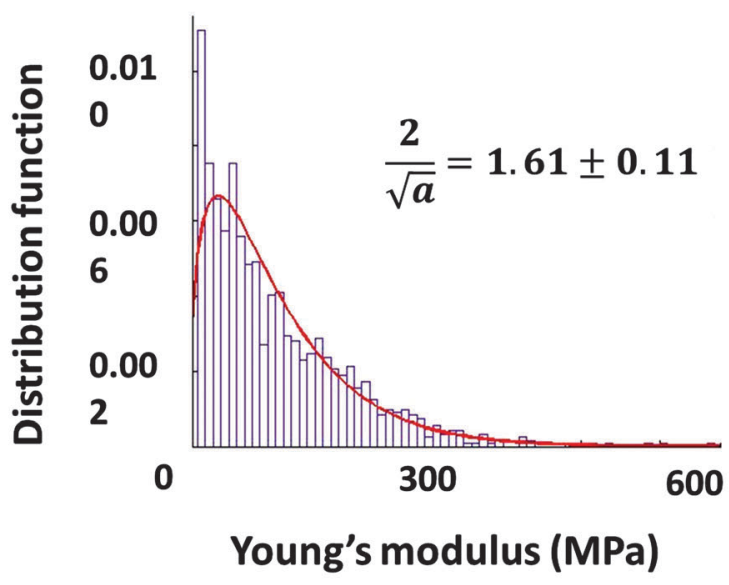

B

Ph-surface

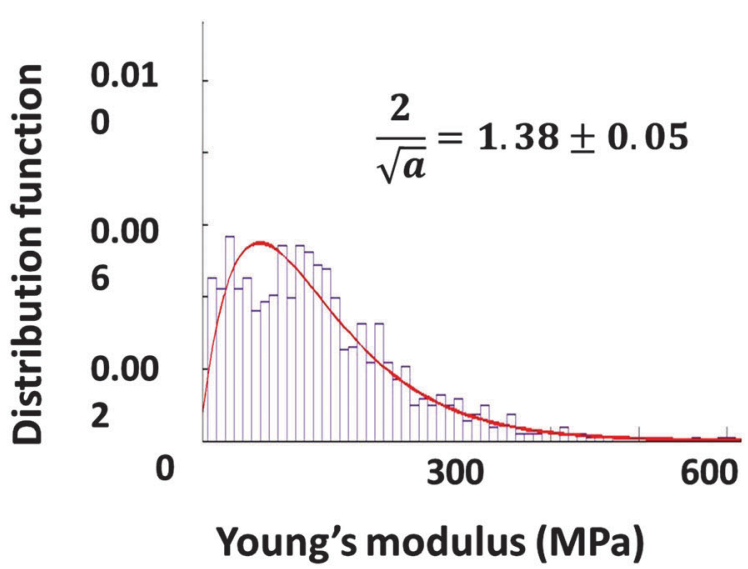

Fig. 4 Histograms of the Young's modulus (determined by AFM) fitted with distribution functions for the $\mathrm{C} 14$-cytidine gel films on the $\mathrm{OH}$-surface (A) and the Ph-surface (B). 
the molecular architecture of the gels. To assess if this effect is restricted to self-assembly processes in the proximity of the modified surface or if it extends further into the bulk of the gels we measured fibre diameters from gels prepared in vials whose surfaces were also modified to display $\mathrm{OH}$ and phenyl groups (Fig. S4 in ESI $\dagger$ ). No significant differences in the fibre diameters were found, indicating that the range of the surface effect may be limited.

To investigate if the different fibre architectures are accompanied by different mechanical properties in the gel films, the relative mechanical properties (Young's modulus) of gels obtained on the two surfaces were measured via nanoindentation with AFM. Nanoindentation measurements can be used for films as thin as $1 \mu \mathrm{m}^{16}$ and are therefore suitable for the present gel films (thickness: $1.0 \pm 0.2 \mu \mathrm{m}$ ).

The data was plotted as histograms and fitted to a gamma distribution $(p$-value $<0.01)$. The distributions of the stiffness measurements and the skewness of the curve fits ( $\alpha$ values) are presented in Fig. 4. The Young's moduli measured on both samples are distributed over the same range (10-600 MPa). The relatively high Young's moduli (values in the $\mathrm{kPa}$ range are typical for solvated gels measured in bulk ${ }^{17}$ ) can be explained by the fact that these gels have been dried and their mechanical properties measured as thin films on solid substrates rather than bulk conditions. The datasets for each surface were compared through the parameter $\alpha$ which is indicative for the skewness of the distribution. An unpaired $t$-test $(p$-value $<0.10)$ showed that the skewness was significantly different $(t=2.696$, $\mathrm{d} f=4$ ) on each surface. These results quantitatively demonstrate a distinct difference in the distribution of stiffness values of gel films obtained on different surfaces, indicating that gel films on Ph-surfaces (more hydrophobic and rougher) are stiffer than those on $\mathrm{OH}$-surfaces (more hydrophilic, smoother).

The effect of different surfaces on supramolecular gel film properties was investigated and quantified for the first time.
We show that the gelator formed gels with different fibre diameter and different gel stiffness. This demonstrates a direct relationship between surface and gel properties, highlighting the importance of surface properties in self-assembly and providing new means for control over gel functionality.

This work was support by the EPSRC funded CDT in Targeted Therapeutics grant EP/L01646X and an RSC research bursary.

\section{References}

1 L. Adler-Abramovich and E. Gazit, Chem. Soc. Rev., 2014, 43, 6881-6893.

2 E. Busseron, Y. Ruff, E. Moulin and N. Giuseppone, Nanoscale, 2013, 5, 7098-7140.

3 K. J. Skilling, F. Citossi, T. D. Bradshaw, M. Ashford, B. Kellam and M. Marlow, Soft Matter, 2014, 10, 237-256.

4 N. M. Sangeetha and U. Maitra, Chem. Soc. Rev., 2005, 34, 821-836.

5 H. Wang, Z. Yang and D. J. Adams, Mater. Today, 2012, 15, 500-507.

6 R. J. Williams, R. J. Mart and R. V. Ulijn, Pept. Sci., 2010, 94, 107-117.

7 R. Huang, R. Su, W. Qi, J. Zhao and Z. He, Nanotechnology, 2011, 22, 245609 .

8 J. J. Gooding, F. Mearns, W. Yang and J. Liu, Electroanalysis, 2003, 15, 81-96.

9 M. O. Blunt, J. C. Russell, M. del Carmen Gimenez-Lopez, N. Taleb, X. Lin, M. Schröder, N. R. Champness and P. H. Beton, Nat. Chem., 2011, 3, 74-78.

10 R. J. Williams, A. M. Smith, R. Collins, N. Hodson, A. K. Das and R. V. Ulijn, Nat. Nanotechnol., 2009, 4, 19-24.

11 E. K. Johnson, L. Chen, P. S. Kubiak, S. F. McDonald, D. J. Adams and P. J. Cameron, Chem. Commun., 2013, 49, 8698-8700.

12 S. S. Lee, S. B. Tang, D. M. Smilgies, A. R. Woll, M. A. Loth, J. M. Mativetsky, J. E. Anthony and Y. L. Loo, Adv. Mater., 2012, 24, 2692-2698.

13 R. G. Weiss, J. Am. Chem. Soc., 2014, 136, 7519-7530.

14 K. Skilling, A. Ndungu, B. Kellam, M. Ashford, T. Bradshaw and M. Marlow, J. Mater. Chem. B, 2014, 2, 8412-8417.

15 X. Dong, A. Gusev and D. M. Hercules, J. Am. Soc. Mass Spectrom., 1998, 9, 292-298.

16 J. Domke and M. Radmacher, Langmuir, 1998, 14, 3320-3325.

17 M. Oyen, Int. Mater. Rev., 2014, 59, 44-59. 